\title{
IDENTITIES FOR 3-CORE AND 5-CORE PARTITIONS
}

\author{
YOON KYUng PARK
}

\begin{abstract}
We apply modular function theory to find the relation among $t$-core partitions. By using the generators of function field corresponding to a certain modular group, we reprove the identities in [1] because their relations are linear for $t=3$ or 5 .
\end{abstract}

\section{Introduction}

For a positive integer $n$, a partition of $n$ is a non-increasing sequence of positive integers whose sum is $n$. Let $p(n)$ be the number of partitions of $n$ with the following generating function:

$$
\sum_{n=0}^{\infty} p(n) q^{n}=\prod_{n=1}^{\infty} \frac{1}{1-q^{n}}=\frac{q^{-\frac{1}{24}}}{\eta(\tau)},
$$

where $\tau$ is in the complex upper half plane $\mathfrak{H}, \eta(\tau)$ is Dedekind's eta function and $q=e^{2 \pi i \tau}$. In a symmetric group $S_{n}$, a conjugacy class is the set of permutations with a given cycle structure, and so they are in a natural 1-1 correspondence with the set of partitions of $n$.

There are various partition functions to give a condition to each part. One of them is $t$-core partition.

The Ferres-Young diagram of a partition $\lambda$ of $n$ is a finite collection of nodes arranged in left-justified rows with having $\lambda_{k}$ nodes in $k$ th row. The hook is the set of nodes directly below, together with the set of nodes directly to the right of, the $(i, j)$ node, as well as the $(i, j)$ node itself. For each node, the hook number $H(i, j)$ is defined as the total number of nodes on the $(i, j)$ hook. A partition $\lambda$ is said to be a $t$-core if it has no hook numbers that are multiples of $t$. For instance, the 3 -core partition $\lambda=(3,1,1)$ of 5 has the Ferres-Young diagram:

- (5) • (2) • (1)

- $(2)$

- $(1)$

Received January 8, 2013; Revised October 30, 2013.

2010 Mathematics Subject Classification. 05A17, 11F03, 11F30.

Key words and phrases. $t$-cores, modular functions. 
Here the number in blank means hook number of each node. This is the only 3 -core partition of 5 .

To introduce generating function of $t$-core partition, for $|q|<1$ we use the standard notation

$$
(a ; q)_{\infty}:=\prod_{n=0}^{\infty}\left(1-a q^{n}\right)
$$

We denote $f(-q)$ by

$$
f(-q):=\sum_{n=-\infty}^{\infty}(-1)^{n} q^{\frac{n(3 n-1)}{2}}=(q ; q)_{\infty}=q^{-\frac{1}{24}} \eta(\tau) .
$$

Let $a_{t}(n)$ be number of partitions of $n$ which are $t$-cores. Then the generating function for $a_{t}(n)$ is given by [5]:

$$
\sum_{n=0}^{\infty} a_{t}(n) q^{n}=\frac{f^{t}\left(-q^{t}\right)}{f(-q)}
$$

With these definitions the followings are our main theorems:

Theorem 1. If $a_{3}(n)$ denotes the number of partitions of $n$ that are 3 -cores, then

$$
a_{3}(4 n+1)=a_{3}(n) .
$$

Theorem 2. Let $a_{5}(n)$ denote the number of partitions of $n$ that are 5-cores. Then

$$
a_{5}(4 n+3)=a_{5}(2 n+1)+2 a_{5}(n) .
$$

Baruah and Berndt proved the above two $t$-core partition identities using the formulas in Ramanujan's notebooks [3] and [10] in 2007 ([1]). Hirschhorn and Sellers generalized Theorem 1 using divisor function and found infinitely many 3 -core partition identities ([6]). Berkovich and Yesilyurt found 7-core identities by the help of Ramanujan's identities like Berndt and Baruah ([2]). After then Kim proved their result using modular form theory ([8]). Here we prove Theorem 1 and 2 by means of modular equations. For two modular functions, there always exists a modular equation by Ishida and Ishii's theorem (Theorem 4), if we find the modular group corresponding to these two functions. Especially, when these functions are generating functions for some partition function, the modular equation just found gives us some relation between partition functions without any appeared identities in other papers or books. Since every $t$-core partition derives its modularity, we always get their relations. Moreover we get simpler form in case $t=3$ and 5 because they are linear.

In $\S 2$ we review a brief modular function theory including Klein forms. We give the proofs of Theorems 1 and 2 in $\S 3$ and $\S 4$, respectively.

Hereafter, we denote the generating functions of $t$-core partitions as follows:

$$
g_{t, 1}(\tau):=g_{t, 1}(q):=\sum_{n=0}^{\infty} a_{t}(n) q^{n}=\frac{f^{t}\left(-q^{t}\right)}{f(-q)},
$$




$$
\begin{aligned}
& g_{t, s}(\tau):=g_{t, s}(q):=\frac{f^{t}\left(-q^{t s}\right)}{f\left(-q^{s}\right)} \text { for an integer } s \geq 1 \\
&\left(:=g_{t, 1}(s \tau)\right)
\end{aligned}
$$

and

$$
g_{t,-1}(\tau):=g_{t,-1}(q):=\frac{f^{t}\left(q^{t}\right)}{f(q)}
$$

\section{Preliminaries}

We would like to state some necessary definitions and properties from the theory of modular functions. Denote $\mathfrak{H}^{*}$ by $\mathfrak{H} \cup \mathbb{Q} \cup\{\infty\}$.

Let $\Gamma(1)=S L_{2}(\mathbb{Z})$ be the full modular group. For any integer $N \geq 1$, we have congruence subgroups $\Gamma(N), \Gamma_{1}(N), \Gamma_{0}(N)$ and $\Gamma^{0}(N)$ of $\Gamma(1)$ consisting of matrices $\left(\begin{array}{ll}a & b \\ c & d\end{array}\right)$ congruent modulo $N$ to $\left(\begin{array}{ll}1 & 0 \\ 0 & 1\end{array}\right),\left(\begin{array}{ll}1 & * \\ 0 & 1\end{array}\right),\left(\begin{array}{ll}* & * \\ 0 & *\end{array}\right)$ and $\left(\begin{array}{ll}* & 0 \\ * & *\end{array}\right)$, respectively. Then a congruence subgroup $\Gamma$ acts on $\mathfrak{H}^{*}$ by linear fractional transformations so that $\gamma(\tau)=(a \tau+b) /(c \tau+d)$ for $\gamma=\left(\begin{array}{ll}a & b \\ c & d\end{array}\right) \in \Gamma$, and the quotient space $\Gamma \backslash \mathfrak{H}^{*}$ becomes a compact Riemann surface with an appropriate complex structure. By definition an element $s$ of $\mathbb{Q} \cup\{\infty\}$ is called a cusp, and two cusps $s_{1}, s_{2}$ are equivalent under $\Gamma$ if there exists $\gamma \in \Gamma$ such that $\gamma\left(s_{1}\right)=s_{2}$. Then the equivalence class of a cusp $s$ or its representative $s$ is called a cusp of $\Gamma$ by abuse of terminology. Indeed, there exist at most finitely many inequivalent cusps of $\Gamma$. Let $s$ be any cusp of $\Gamma$, and let $\rho \in S L_{2}(\mathbb{Z})$ be such that $\rho(s)=\infty$. We define the width of the cusp $s$ in $\Gamma \backslash \mathfrak{H}^{*}$ by the smallest positive integer $h$ satisfying $\rho^{-1}\left(\begin{array}{ll}1 & h \\ 0 & 1\end{array}\right) \rho \in\{ \pm 1\} \cdot \Gamma$. Then the width depends only on the equivalence class of the cusp $s$ under $\Gamma$ and is independent of the choice of $\rho$.

By a modular function with respect to a congruence subgroup $\Gamma$ we mean a $\mathbb{C}$-valued function $f(\tau)$ of $\mathfrak{H}$ satisfying the following three conditions:

(1) $f(\tau)$ is meromorphic on $\mathfrak{H}$,

(2) $f(\tau)$ is invariant under $\Gamma$, i.e., $f \circ \gamma=f$ for all $\gamma \in \Gamma$ and $\tau \in \mathfrak{H}$,

(3) $f(\tau)$ is meromorphic at all cusps of $\Gamma$.

The precise meaning of the last condition is as follows. For a cusp $s$ for $\Gamma$, let $h$ be the width for $s$ and $\rho$ be an element of $S L_{2}(\mathbb{Z})$ such that $\rho(s)=\infty$. Since $\left(f \circ \rho^{-1}\right)(\tau+h)=\left(f \circ \rho^{-1}\left(\begin{array}{cc}1 & h \\ 0 & 1\end{array}\right) \rho\right)\left(\rho^{-1} \tau\right)=\left(f \circ \rho^{-1}\right)(\tau), f \circ \rho^{-1}$ has a Laurent series expansion in $q_{h}=e^{2 \pi i \tau / h}$, namely for some integer $n_{0},\left(f \circ \rho^{-1}\right)(\tau)=$ $\sum_{n \geq n_{0}} a_{n} q_{h}^{n}$ with $a_{n_{0}} \neq 0$. This integer $n_{0}$ is called the order of $f(\tau)$ at the cusp $s$ and denoted by $\operatorname{ord}_{s} f(\tau)$. If $\operatorname{ord}_{s} f(\tau)$ is positive (respectively, negative), then we say that $f(\tau)$ has a zero (respectively, a pole) at $s$. If a modular function $f(\tau)$ is holomorphic on $\mathfrak{H}$ and $\operatorname{ord}_{s} f(\tau)$ is greater than or equal to 0 for every cusp $s$, then we say that $f(\tau)$ is holomorphic on $\mathfrak{H}^{*}$. Since we may identify a modular function with respect to $\Gamma$ with a meromorphic function on the compact Riemann surface $\Gamma \backslash \mathfrak{H}^{*}$, any holomorphic modular function with respect to some congruence subgroup $\Gamma$ is a constant. 
Let $A_{0}(\Gamma)$ be the field of all modular functions with respect to $\Gamma$, and $A_{0}(\Gamma)_{\mathbb{Q}}$ be the subfield of $A_{0}(\Gamma)$ in which the Fourier expansion of $f(\tau)$ has rational coefficients. Then we may identify $A_{0}(\Gamma)$ with the field $\mathbb{C}\left(\Gamma \backslash \mathfrak{H}^{*}\right)$ of all meromorphic functions of the compact Riemann surface $\Gamma \backslash \mathfrak{H}^{*}$, and if $f(\tau) \in A_{0}(\Gamma)$ is nonconstant, then the field extension degree $\left[A_{0}(\Gamma): \mathbb{C}(f(\tau))\right]$ is finite and is equal to the total degree of poles of $f(\tau)$. Since we will consider the modular functions with neither zeros nor poles on $\mathfrak{H}$, the total degree of poles of $f(\tau)$ is $-\Sigma_{s} \operatorname{ord}_{s} f(\tau)$ where the summation runs over all the inequivalent cusps $s$ at which $f(\tau)$ has poles.

From now on we briefly review the Klein forms, which are mainly used in this paper. We refer to [9] for more details. For any lattice $L \subset \mathbb{C}$ and $z \in \mathbb{C}$, we define the Weierstrass $\sigma$-function by

$$
\sigma(z ; L)=z \prod_{\omega \in L\{0\}}\left(1-\frac{z}{\omega}\right) e^{\frac{z}{\omega}+\frac{1}{2}\left(\frac{z}{\omega}\right)^{2}}
$$

which is holomorphic with only simple zeros at all points $z \in L$. We further define the Weierstrass $\zeta$-function by the logarithmic derivative of the Weierstrass $\sigma$-function, i.e.,

$$
\zeta(z ; L)=\frac{\sigma^{\prime}(z ; L)}{\sigma(z ; L)}=\frac{1}{z}+\sum_{z \in L-\{0\}}\left(\frac{1}{z-\omega}+\frac{1}{\omega}+\frac{z}{\omega^{2}}\right)
$$

which is meromorphic with only simple poles at all points $z \in L$. It is easy to see that the Weierstrass $\sigma$-function (respectively, the Weierstrass $\zeta$-function) is homogeneous of degree 1 (respectively, -1), that is, $\sigma(\lambda z ; \lambda L)=\lambda \sigma(z ; L)$ (respectively, $\left.\zeta(\lambda z ; \lambda L)=\lambda^{-1} \zeta(z ; L)\right)$ for any $\lambda \in \mathbb{C}^{\times}$. Note that $\zeta^{\prime}(z ; L)=$ $-\wp(z ; L)$ where

$$
\wp(z ; L)=\frac{1}{z^{2}}+\sum_{\omega \in L-\{0\}}\left(\frac{1}{(z-\omega)^{2}}-\frac{1}{\omega^{2}}\right)
$$

is the Weierstrass $\wp$-function. Since the Weierstrass $\wp$-function is an elliptic function, namely $\wp(z+\omega ; L)=\wp(z ; L)$ for $\omega \in L$, we obtain that $\frac{d}{d z}(\zeta(z+\omega ; L)-$ $\zeta(z ; L))=0$ for any $\omega \in L$. This means that $\zeta(z+\omega ; L)-\zeta(z ; L)$ depends only on $\omega \in L$, not on $z \in \mathbb{C}$. Thus we may define $\eta(\omega ; L)=\zeta(z+\omega ; L)-\zeta(z ; L)$ for all $\omega \in L$. Let $L=\mathbb{Z} \omega_{1}+\mathbb{Z} \omega_{2}$. For $z=a_{1} \omega_{1}+a_{2} \omega_{2}$ with $a_{1}, a_{2} \in \mathbb{R}$ we define the Weierstrass $\eta$-function by

$$
\eta(z ; L)=a_{1} \eta\left(\omega_{1} ; L\right)+a_{2} \eta\left(\omega_{2} ; L\right) .
$$

Then it is easy to see that the Weierstrass $\eta$-function $\eta(z ; L)$ is well-defined, in other words it does not depend on the choice of the basis $\left\{\omega_{1}, \omega_{2}\right\}$ of $L$, and $\eta(z ; L)$ is $\mathbb{R}$-linear so that $\eta(r z ; L)=r \eta(z ; L)$ for any $r \in \mathbb{R}$. Note that since the Weierstrass $\zeta$-function is homogeneous of degree -1 , so is the Weierstrass $\eta$-function. We define the Klein form by

$$
\mathfrak{k}(z ; L)=e^{-\eta(z ; L) z / 2} \sigma(z ; L) .
$$


Let $\mathbf{a}=\left(a_{1} a_{2}\right) \in \mathbb{R}^{2}$ and $\tau \in \mathfrak{H}$. We further define

$$
\mathfrak{k}_{\mathbf{a}}(\tau)=\mathfrak{k}\left(a_{1} \tau+a_{2} ; \mathbb{Z} \tau+\mathbb{Z}\right)
$$

which is also called the Klein form by abuse of terminology. Here we observe that $\mathfrak{k}_{\mathbf{a}}(\tau)$ is holomorphic and nonvanishing on $\mathfrak{H}$ if $\mathbf{a} \in \mathbb{R}^{2}-\mathbb{Z}^{2}$ and that the Klein form is homogeneous of degree 1 , i.e., $\mathfrak{k}(\lambda z ; \lambda L)=\lambda \mathfrak{k}(z ; L)$.

The Klein form satisfies the following properties (see [9]). Let $\gamma=\left(\begin{array}{ll}a & b \\ c & d\end{array}\right) \in$ $S L_{2}(\mathbb{Z})$ and $\mathbf{a}=\left(a_{1} a_{2}\right) \in \mathbb{R}^{2}-\mathbb{Z}^{2}$.

(K0) $\mathfrak{k}_{-\mathbf{a}}(\tau)=-\mathfrak{k}_{\mathbf{a}}(\tau)$.

(K1) $\mathfrak{k}_{\mathbf{a}}(\gamma(\tau))=(c \tau+d)^{-1} \mathfrak{k}_{\mathbf{a} \gamma}(\tau)$.

(K2) For any $\mathbf{b}=\left(\begin{array}{ll}b_{1} & b_{2}\end{array}\right) \in \mathbb{Z}^{2}$ we have

$$
\mathfrak{k}_{\mathbf{a}+\mathbf{b}}(\tau)=\varepsilon(\mathbf{a}, \mathbf{b}) \mathfrak{k}_{\mathbf{a}}(\tau)
$$

where $\varepsilon(\mathbf{a}, \mathbf{b})=(-1)^{b_{1} b_{2}+b_{1}+b_{2}} e^{\pi i\left(b_{2} a_{1}-b_{1} a_{2}\right)}$.

(K3) For $\mathbf{a}=\left(\frac{r}{N} \frac{s}{N}\right) \in \frac{1}{N} \mathbb{Z}^{2}-\mathbb{Z}^{2}$ and any $\gamma \in \Gamma(N)$ with an integer $N>1$,

$$
\mathfrak{k}_{\mathbf{a}}(\gamma(\tau))=\varepsilon_{\mathbf{a}}(\gamma) \cdot(c \tau+d)^{-1} \cdot \mathfrak{k}_{\mathbf{a}}(\tau),
$$

where

$$
\varepsilon_{\mathbf{a}}(\gamma)=-(-1)^{\left(\frac{a-1}{N} r+\frac{c}{N} s+1\right)\left(\frac{b}{N} r+\frac{d-1}{N} s+1\right)} \cdot e^{\pi i\left(b r^{2}+(d-a) r s-c s^{2}\right) / N^{2}} .
$$

(K4) Let $\tau \in \mathfrak{H}, z=a_{1} \tau+a_{2}$ with $\mathbf{a}=\left(a_{1} a_{2}\right) \in \mathbb{Q}^{2}-\mathbb{Z}^{2}$, and further let $q=e^{2 \pi i \tau}, q_{z}=e^{2 \pi i z}=e^{2 \pi i a_{2}} e^{2 \pi i a_{1} \tau}$. Then

$$
\mathfrak{k}_{\mathbf{a}}(\tau)=-\frac{1}{2 \pi i} e^{\pi i a_{2}\left(a_{1}-1\right)} \cdot q^{\frac{1}{2} a_{1}\left(a_{1}-1\right)} \cdot\left(1-q_{z}\right) \cdot \prod_{n=1}^{\infty} \frac{\left(1-q^{n} q_{z}\right)\left(1-q^{n} q_{z}^{-1}\right)}{\left(1-q^{n}\right)^{2}}
$$

and $\operatorname{ord}_{q} \mathfrak{k}_{\mathbf{a}}(\tau)=\frac{1}{2}\left\langle a_{1}\right\rangle\left(\left\langle a_{1}\right\rangle-1\right)$ where $\left\langle a_{1}\right\rangle$ denotes the number such that $0 \leq\left\langle a_{1}\right\rangle<1$ and $a_{1}-\left\langle a_{1}\right\rangle \in \mathbb{Z}$.

(K5) Let $f(\tau)=\prod_{\mathbf{a}} \mathfrak{k}_{\mathbf{a}}^{m(\mathbf{a})}(\tau)$ be a finite product of Klein forms with $\mathbf{a}=$ $\left(\frac{r}{N} \frac{s}{N}\right) \in \frac{1}{N} \mathbb{Z}^{2}-\mathbb{Z}^{2}$ for an integer $N>1$, and let $k=-\sum_{\mathbf{a}} m(\mathbf{a})$. Then $f(\tau)$ is a modular function with respect to $\Gamma(N)$ if and only if $k=0$ and $\left\{\sum_{\mathbf{a}} m(\mathbf{a}) r^{2} \equiv \sum_{\mathbf{a}} m(\mathbf{a}) s^{2} \equiv \sum_{\mathbf{a}} m(\mathbf{a}) r s \equiv 0 \bmod N\right.$ if $N$ is odd

$\left\{\sum_{\mathbf{a}} m(\mathbf{a}) r^{2} \equiv \sum_{\mathbf{a}} m(\mathbf{a}) s^{2} \equiv 0 \bmod 2 N, \sum_{\mathbf{a}} m(\mathbf{a}) r s \equiv 0 \bmod N\right.$ if $N$ is even.

For positive integers $N$ and $r$ with $0 \leq r \leq N-1$, we write

$$
K_{\frac{r}{N}}(\tau)=\prod_{j=0}^{N-1} \mathfrak{k}_{\left(\frac{r}{N}, \frac{j}{N}\right)}(\tau) .
$$

By the help of the property (K2), for $0 \leq r \leq\left[\frac{N}{2}\right]$ the functions $K_{\frac{r}{N}}$ are enough to derive our results. 
Before closing this section, we introduce the useful statements giving equations among generating functions for $t$-core partitions.

A complete set $S_{\Gamma_{0}(N)}$ of representatives for the cusps of $\Gamma_{0}(N)$ is

$$
\begin{aligned}
& S_{\Gamma_{0}(N)}=\left\{\frac{a_{c}}{c} \in \mathbb{Q}: c \mid N, 1 \leq a_{c} \leq N, \operatorname{gcd}\left(a_{c}, N\right)\right.=1, \\
&\left.a_{c} \equiv a_{c}^{\prime}(\bmod \operatorname{gcd}(c, N / c)) \Leftrightarrow a_{c}=a_{c}^{\prime}\right\},
\end{aligned}
$$

and the number of inequivalent cusps of $\Gamma_{0}(N)$ is

$$
\left|S_{\Gamma_{0}(N)}\right|=\sum_{\substack{c>0 \\ c \mid N}} \frac{\phi(c) \phi(N / c)}{\phi\left(\frac{N}{\operatorname{gcd}(c, N / c)}\right)}
$$

where $\phi(k)$ is Euler phi-function.

Since $\left(\begin{array}{cc}1 & 0 \\ N & 1\end{array}\right) \in \Gamma_{0}(N)$ we can consider $\infty$ as $\frac{1}{N}$ and we discuss the width at this cusp.

Lemma 3. Let $\frac{a}{c}$ be a cusp of $\Gamma_{0}(N)$ with $a, c \in \mathbb{Z},(a, c)=1$ and $0<c \mid N$. We understand $\frac{ \pm 1}{0}$ as $\infty$ equivalent to $\frac{1}{N}$. Then the width $h$ of a cusp $\frac{a}{c}$ in $\Gamma_{0}(N) \backslash \mathfrak{H}^{*}$ is

$$
h=\frac{N}{\left(N, c^{2}\right)} .
$$

Proof. Note that the width $h$ of cusp $\frac{a}{c}$ in $\Gamma_{0}(N) \backslash \mathfrak{H}^{*}$ is the smallest positive integer satisfying

$$
\left(\begin{array}{ll}
a & b \\
c & d
\end{array}\right)\left(\begin{array}{ll}
1 & h \\
0 & 1
\end{array}\right)\left(\begin{array}{ll}
a & b \\
c & d
\end{array}\right)^{-1}=\left(\begin{array}{cc}
* & * \\
-c^{2} h & *
\end{array}\right) \in\{ \pm 1\} \cdot \Gamma_{0}(N) .
$$

Hence $c^{2} h$ should be a multiple of $N$ and $\frac{N}{\left(N, c^{2}\right)}$ is the smallest positive integer such $h$.

For the result of the general subgroup instead of $\Gamma_{0}(N)$, we may refer to [4]. Let $F(X, Y)$ be a polynomial such that

$$
F(X, Y)=\sum_{i, j} C_{i, j} X^{i} Y^{j} \in \mathbb{Q}[X, Y]
$$

and $F\left(f_{1}(\tau), f_{2}(\tau)\right)=0$ for some two modular forms $f_{1}$ and $f_{2}$. The next theorem is useful in knowing which coefficients of the modular equations are zero in $F_{n}(X, Y)$.

Theorem $4([7])$. For any congruence subgroup $\Gamma^{\prime}$, let $f_{1}(\tau), f_{2}(\tau)$ be nonconstants such that $\mathbb{C}\left(f_{1}(\tau), f_{2}(\tau)\right)=A_{0}\left(\Gamma^{\prime}\right)$ with the total degree $D_{k}$ of poles of $f_{k}(\tau)$ for $k=1,2$, and let

$$
F(X, Y)=\sum_{\substack{0 \leq i \leq D_{2} \\ 0 \leq j \leq D_{1}}} C_{i, j} X^{i} Y^{j} \in \mathbb{C}[X, Y]
$$


be such that $F\left(f_{1}(\tau), f_{2}(\tau)\right)=0$. Let $S_{\Gamma^{\prime}}$ be a set of all the inequivalent cusps of $\Gamma^{\prime}$, and for $k=1,2$,

$$
S_{k, 0}=\left\{s \in S_{\Gamma^{\prime}} \mid f_{k}(\tau) \text { has zeros at } s\right\},
$$

and

$$
S_{k, \infty}=\left\{s \in S_{\Gamma^{\prime}} \mid f_{k}(\tau) \text { has poles at } s\right\}
$$

Further let

$$
a= \begin{cases}0, & \text { if } S_{1, \infty} \cap S_{2,0}=\phi \\ -\sum_{s \in S_{1, \infty} \cap S_{2,0}} \operatorname{ord}_{s} f_{1}(\tau), & \text { otherwise }\end{cases}
$$

and

$$
b= \begin{cases}0, & \text { if } S_{1,0} \cap S_{2,0}=\phi \\ \sum_{s \in S_{1,0} \cap S_{2,0}} \operatorname{ord}_{s} f_{1}(\tau), & \text { otherwise. }\end{cases}
$$

Then we obtain the following assertions.

(1) $C_{D_{2}, a} \neq 0$. If further $S_{1, \infty} \subset S_{2, \infty} \cup S_{2,0}$, then $C_{D_{2}, j}=0$ for any $j \neq a$.

(2) $C_{0, b} \neq 0$. If further $S_{1,0} \subset S_{2, \infty} \cup S_{2,0}$, then $C_{0, j}=0$ for any $j \neq b$.

(3) $C_{i, D_{1}}=0$ for all $i$ satisfying $0 \leq i<\left|S_{1,0} \cap S_{2, \infty}\right|$ or $D_{2}-\left|S_{1, \infty} \cap S_{2, \infty}\right|$ $<i \leq D_{2}$.

(4) $C_{i, 0}=0$ for all $i$ satisfying $0 \leq i<\left|S_{1,0} \cap S_{2,0}\right|$ or $D_{2}-\left|S_{1, \infty} \cap S_{2,0}\right|<$ $i \leq D_{2}$.

If we interchange the roles of $f_{1}(\tau)$ and $f_{2}(\tau)$, then we may obtain further properties similar to $(1) \sim(4)$. Suppose further that there exist $r \in \mathbb{R}$ and $N, n_{1}, n_{2} \in \mathbb{Z}$ with $N>0$ such that $f_{k}(\tau+r)=\zeta_{N}^{n_{k}} f_{k}(\tau)$ for $k=1,2$, where $\zeta_{N}=e^{2 \pi i / N}$. Then we obtain the following assertion.

(5) $n_{1} i+n_{2} j \not n_{1} D_{2}+n_{2} a \bmod N \Rightarrow C_{i, j}=0$. Here note that $n_{2} b \equiv$ $n_{1} D_{2}+n_{2} a \bmod N$.

Proof. We refer to [7].

\section{Proof of Theorem 1}

Let $g_{3, s}(\tau)$ be the modified generating function of 3-core partition defined in $\S 1$. Hereafter, we denote $h_{1}^{(3)}(\tau)$ (resp. $\left.h_{4}^{(3)}(\tau)\right)$ by $\frac{g_{3,1}}{g_{3,-1}}(\tau)$ (resp. $\left.q \frac{g_{3,4}}{g_{3,-1}}(\tau)\right)$.

Lemma 5. The function $h_{1}^{(3)}(\tau)$ and $h_{4}^{(3)}(\tau)$ can be written as the following Klein form quotients.

(1) $h_{1}^{(3)}(\tau)=\frac{g_{3,1}}{g_{3,-1}}(\tau)=\zeta_{12}^{-1} \frac{K_{\frac{2}{12}} K_{\frac{3}{12}}^{4}}{K_{\frac{1}{12}}^{2} K_{\frac{5}{12}}^{2} K_{\frac{6}{12}}}(\tau)$,

(2) $h_{4}^{(3)}(\tau)=q \frac{g_{3,4}}{g_{3,-1}}(\tau)=\zeta_{6}^{-1} \frac{K_{\frac{2}{12}}^{2} K_{\frac{3}{12}}^{2}}{K_{\frac{1}{12}} K_{\frac{5}{12}} K_{\frac{6}{12}}^{2}}(\tau)$. 
Proof. Observe the functions $K_{\frac{r}{12}}(r=1,2, \ldots, 11)$. (K4) means that

$$
\begin{aligned}
& K_{\frac{r}{12}}(\tau) \\
= & \prod_{j=0}^{11} \mathfrak{k}_{\left(\frac{r}{12}, \frac{j}{12}\right)}(\tau) \\
= & (-2 \pi i)^{-12} \zeta_{48}^{11 r+12} q^{\frac{r(r-12)}{24}} \prod_{j=0}^{11}\left(1-\zeta_{12}^{j} q^{\frac{r}{12}}\right) \prod_{n=1}^{\infty} \frac{\left(1-\zeta_{12}^{j} q^{n+\frac{r}{12}}\right)\left(1-\zeta_{12}^{-j} q^{n-\frac{r}{12}}\right)}{\left(1-q^{n}\right)^{2}} \\
= & (-2 \pi i)^{-12} \zeta_{48}^{11 r+12} q^{\frac{r(r-12)}{24}} \prod_{n=1}^{\infty} \frac{\left(1-q^{12 n-(12-r)}\right)\left(1-q^{12 n-r}\right)}{\left(1-q^{n}\right)^{24}}
\end{aligned}
$$

and (1.1) implies the followings:

$$
\begin{aligned}
& h_{1}^{(3)}(\tau) \\
= & \prod_{n=1}^{\infty} \frac{\left(1-q^{3 n}\right)^{3}}{\left(1-q^{n}\right)} / \frac{\left(1-(-q)^{3 n}\right)^{3}}{\left(1-(-q)^{n}\right)} \\
= & \prod_{n=1}^{\infty} \frac{\left(1-q^{12 n-2}\right)\left(1-q^{12 n-10}\right)\left(1-q^{12 n-3}\right)^{4}\left(1-q^{12 n-9}\right)^{4}}{\left(1-q^{12 n-1}\right)^{2}\left(1-q^{12 n-11}\right)^{2}\left(1-q^{12 n-5}\right)^{2}\left(1-q^{12 n-7}\right)^{2}\left(1-q^{12 n-6}\right)^{2}},
\end{aligned}
$$

and

$$
\begin{aligned}
h_{4}^{(3)}(\tau) & =\prod_{n=1}^{\infty} \frac{\left(1-q^{12 n}\right)^{3}}{\left(1-q^{4 n}\right)} / \frac{\left(1-(-q)^{3 n}\right)^{3}}{\left(1-(-q)^{n}\right)} \\
& =\prod_{n=1}^{\infty} \frac{\left(1-q^{12 n-2}\right)^{2}\left(1-q^{12 n-10}\right)^{2}\left(1-q^{12 n-3}\right)^{2}\left(1-q^{12 n-9}\right)^{2}}{\left(1-q^{12 n-1}\right)\left(1-q^{12 n-11}\right)\left(1-q^{12 n-5}\right)\left(1-q^{12 n-7}\right)\left(1-q^{12 n-6}\right)^{4}} .
\end{aligned}
$$

Hence by comparing their $q$-products we get the Klein form quotients for $h_{1}^{(3)}(\tau)$ and $h_{4}^{(3)}(\tau)$.

Proposition 6. Let $h_{1}^{(3)}(\tau)$ and $h_{4}^{(3)}(\tau)$ be the functions defined as before. Then the modular function field with respect to $\Gamma_{0}(12)$ is generated by $h_{1}^{(3)}(\tau)$ and $h_{4}^{(3)}(\tau)$ i.e.,

$$
A_{0}\left(\Gamma_{0}(12)\right)=\mathbb{C}\left(h_{1}^{(3)}(\tau), h_{4}^{(3)}(\tau)\right) .
$$

Proof. Assume that $\Gamma^{\prime}$ be the congruence subgroup such that

$$
A_{0}\left(\Gamma^{\prime}\right)=\mathbb{C}\left(h_{1}^{(3)}(\tau), h_{4}^{(3)}(\tau)\right) .
$$

First we show that $\Gamma_{0}(12) \subset \Gamma^{\prime}$. By $($ K5 $)$ and Lemma $5, \Gamma^{\prime}$ is a congruence subgroup of level 12. Furthermore both functions have $q$-expansion, they are in $A_{0}\left(\Gamma_{1}(N)\right)$ because $\Gamma_{1}(N)=\left\langle\Gamma_{0}(N),\left(\begin{array}{ll}1 & 1 \\ 0 & 1\end{array}\right)\right\rangle$. Note that $\left[\overline{\Gamma_{0}(12)}: \overline{\Gamma_{1}(12)}\right]=$ 
$\phi(12) / 2=2$ and $\gamma_{3}=\left(\begin{array}{cc}5 & 2 \\ 12 & 5\end{array}\right)$ is a nontrivial element of $\overline{\Gamma_{0}(12)} / \overline{\Gamma_{1}(12)}$. Observe $\frac{g_{3,1}}{g_{3,-1}}(\tau)$ and $q \frac{g_{3,4}}{g_{3,-1}}(\tau)$,

$$
\begin{aligned}
& K_{\frac{1}{12}}\left(\gamma_{3} \tau\right)=\zeta_{12}(12 \tau+5)^{-12} K_{\frac{5}{12}}(\tau), K_{\frac{5}{12}}\left(\gamma_{3} \tau\right)=\zeta_{12}^{-1}(12 \tau+5)^{-12} K_{\frac{1}{12}}(\tau) \\
& K_{\frac{2}{12}}\left(\gamma_{3} \tau\right)=\zeta_{4}(12 \tau+5)^{-12} K_{\frac{2}{12}}(\tau), K_{\frac{3}{12}}\left(\gamma_{3} \tau\right)=\zeta_{6}^{-1}(12 \tau+5)^{-12} K_{\frac{3}{12}}(\tau)
\end{aligned}
$$

and

$$
K_{\frac{4}{12}}\left(\gamma_{3} \tau\right)=\zeta_{4}^{-1}(12 \tau+5)^{-12} K_{\frac{4}{12}}(\tau), K_{\frac{6}{12}}\left(\gamma_{3} \tau\right)=\zeta_{12}^{7}(12 \tau+5)^{-12} K_{\frac{6}{12}}(\tau)
$$

Hence

$$
\begin{aligned}
h_{1}^{(3)}\left(\gamma_{3} \tau\right) & =\zeta_{12}^{-1} \frac{K_{\frac{2}{12}} K_{\frac{3}{12}}^{4}}{K_{\frac{1}{12}}^{2} K_{\frac{5}{12}}^{2} K_{\frac{6}{12}}}\left(\gamma_{3} \tau\right) \\
& =\zeta_{12}^{-1} \frac{\zeta_{4} \cdot\left(\zeta_{6}^{-1}\right)^{4}}{\left(\zeta_{12}\right)^{2}\left(\zeta_{12}^{-1}\right)^{2} \zeta_{12}^{7}} \cdot \frac{K_{\frac{2}{12}} K_{\frac{3}{12}}^{4}}{K_{\frac{1}{12}}^{2} K_{\frac{5}{12}}^{2} K_{\frac{6}{12}}}(\tau) \\
& =h_{1}^{(3)}(\tau)
\end{aligned}
$$

and

$$
\begin{aligned}
h_{4}^{(3)}\left(\gamma_{3} \tau\right) & =\zeta_{6}^{-1} \frac{K_{\frac{2}{12}}^{2} K_{\frac{3}{12}}^{2}}{K_{\frac{1}{12}} K_{\frac{5}{12}} K_{\frac{6}{12}}^{2}}\left(\gamma_{3} \tau\right) \\
& =\zeta_{6}^{-1} \frac{\left(\zeta_{4}\right)^{2} \cdot\left(\zeta_{6}^{-1}\right)^{2}}{\zeta_{12} \cdot \zeta_{12}^{-1} \cdot\left(\zeta_{12}^{7}\right)^{2}} \cdot \frac{K_{\frac{2}{12}} K_{\frac{3}{12}}^{4}}{K_{\frac{1}{12}}^{2} K_{\frac{5}{12}}^{2} K_{\frac{6}{12}}}(\tau) \\
& =h_{4}^{(3)}(\tau) .
\end{aligned}
$$

So, $\Gamma_{0}(12) \subset \Gamma^{\prime}$.

For the converse see the behaviors of $h_{1}^{(3)}(\tau)$ and $h_{4}^{(3)}(\tau)$ at each cusp for $\Gamma_{0}(12)$. The following table gives us the exponents of the leading terms of $q$-expansions at each cusp.

\begin{tabular}{c||c|c|c|c|c|c}
\hline cusp & $\infty$ & 0 & $\frac{1}{2}$ & $\frac{1}{3}$ & $\frac{1}{4}$ & $\frac{1}{6}$ \\
\hline$K_{\frac{1}{12}}$ & $-\frac{11}{24}$ & $-\frac{143}{144}$ & $-\frac{73}{72}$ & $-\frac{49}{48}$ & $-\frac{73}{72}$ & $-\frac{23}{24}$ \\
$K_{\frac{2}{12}}$ & $-\frac{5}{6}$ & $-\frac{143}{144}$ & $-\frac{35}{36}$ & $-\frac{49}{48}$ & $-\frac{19}{18}$ & $-\frac{13}{12}$ \\
$K_{\frac{3}{12}}$ & $-\frac{9}{8}$ & $-\frac{143}{144}$ & $-\frac{73}{72}$ & $-\frac{15}{16}$ & $-\frac{73}{72}$ & $-\frac{9}{8}$ \\
$K_{\frac{4}{12}}$ & $-\frac{4}{3}$ & $-\frac{143}{144}$ & $-\frac{35}{36}$ & $-\frac{49}{48}$ & $-\frac{8}{9}$ & $-\frac{13}{12}$ \\
$K_{\frac{5}{12}}$ & $-\frac{35}{24}$ & $-\frac{143}{144}$ & $-\frac{73}{72}$ & $-\frac{49}{48}$ & $-\frac{73}{72}$ & $-\frac{23}{24}$ \\
$K_{\frac{6}{12}}$ & $-\frac{3}{2}$ & $-\frac{143}{144}$ & $-\frac{35}{36}$ & $-\frac{15}{16}$ & $-\frac{19}{18}$ & $-\frac{3}{4}$ \\
\hline$h_{1}^{(3)}$ & 0 & 0 & 0 & $\frac{1}{4}$ & 0 & -1 \\
$h_{4}^{(3)}$ & 1 & 0 & 0 & 0 & 0 & -1 \\
\hline
\end{tabular}


In the above table, for function $f(\tau)$ and the cusp $\frac{a}{c}$ the number at corresponding entry to $f(\tau)$ and $\frac{a}{c}$ means a rational number $r$ satisfying

$$
f\left(\rho^{-1} \tau\right)=q^{r}+\cdots
$$

where $\rho^{-1}=\left(\begin{array}{l}a * \\ c\end{array} *\right) \in S L_{2}(\mathbb{Z})$ and $\rho\left(\frac{a}{c}\right)=\infty$ without considering a width at $\frac{a}{c}$.

Suppose that $\Gamma_{0}(12)$ is a proper subgroup of $\Gamma^{\prime}$. Then there exists an element $\sigma \in \Gamma^{\prime} \backslash \Gamma_{0}(12)$ such that $\sigma(0)=\frac{1}{2}$ and $\sigma$ must be $\left(\begin{array}{cc}a & 1 \\ 2 a-1 & 2\end{array}\right)$ for some $a$.

If $\operatorname{gcd}(2 a-1,12)=1$, then there exist $m$ and $s \in \mathbb{Z}$ such that $(2 a-1) \cdot s+$ $12 a \cdot m=1$ because $\operatorname{gcd}(a, 2 a-1)=1 .\left(\begin{array}{cc}2 a-1 & -a \\ 12 m & s\end{array}\right) \in \Gamma_{0}(12) \subset \Gamma^{\prime}$ means that

$$
\left(\begin{array}{cc}
2 a-1 & -a \\
12 m & s
\end{array}\right) \sigma(\infty)=\left(\begin{array}{cc}
0 & -1 \\
1 & 12 m+s
\end{array}\right)(\infty)=0 .
$$

Hence $\infty$ is equivalent to 0 under $\Gamma^{\prime}$. It is a contradiction.

If $\operatorname{gcd}(2 a-1,12)=3$, then let $k$ be the integer such that $2 a-1=6 k-3$. Since $\operatorname{gcd}(3 k-1,6 k-3)=\operatorname{gcd}(a, 2 a-1)=1$ we can choose integers $s$ and $n$ satisfying $(3 k-1) s-(6 k-3) n=1$ with $s \equiv 2 k-1 \bmod 4$. For an even integer $s$, it is possible to replace $s$ with $s+6 k+3$ because $(3 k-1)(s+6 k-3)-$ $(6 k-3)(n+3 k-1)=1$. For an odd integer $s$ satisfying $s \equiv 2 k-1+2 \bmod 4$ choose $s+12 k-6$ because $(3 k-1)(s+12 k-6)-(6 k-3)(n+6 k-2)=1$. Since $6 k-3-3 s=3(2 k-1-s)$ is a multiple of 12 put $12 N=6 k-3-3 s$ and $r=3 k-3 n-1$. We get $r \cdot s-12 N \cdot n=(3 k-1) s-(6 k-3) n=1$ and $\left(\begin{array}{cc}r & n \\ 12 N & s\end{array}\right) \in \Gamma^{\prime}$. Because

$$
\infty=\sigma^{-1}\left(\frac{a}{2 a-1}\right)=\sigma^{-1}\left(\frac{3 k-1}{6 k-3}\right)=\sigma^{-1}\left(\left(\begin{array}{cc}
r & n \\
12 N & s
\end{array}\right) \frac{1}{3}\right),
$$

$\frac{1}{3}$ and $\infty$ are equivalent cusp in $\Gamma^{\prime}$ and it is a contradiction. Therefore $\Gamma^{\prime}=$ $\Gamma_{0}(12)$.

Then by Theorem 4, we get the equations.

Proof of Theorem 1. Before applying $h_{1}^{(3)}(\tau)$ and $h_{4}^{(3)}(\tau)$ to Theorem 4 we will check where these two functions have pole or zero. Fortunately every Klein forms have pole and zero only at cusps and all we need to know are behaviors of products of Klein forms $K_{\frac{r}{12}}$ at cusps of $\Gamma_{0}(12)$. First we find the inequivalent cusps for $\Gamma_{0}(12)$. By help of (2.2) its inequivalent cusps are $0, \frac{1}{2}, \frac{1}{3}, \frac{1}{4}, \frac{1}{6}$ and $\infty$. Using (K4) we get the table in the proof of Proposition 6 as order of $K_{\frac{r}{12}}$ for $1 \leq r \leq 6$.

Since the widths of cusps are followings:

\begin{tabular}{c||c|c|c|c|c|c}
\hline cusp & $\infty$ & 0 & $\frac{1}{2}$ & $\frac{1}{3}$ & $\frac{1}{4}$ & $\frac{1}{6}$ \\
\hline width & 1 & 12 & 3 & 4 & 3 & 1 \\
\hline
\end{tabular}

the total degrees of poles of both $h_{1}^{(3)}(\tau)$ and $h_{4}^{(3)}(\tau)$ are 1 . Therefore there exists an equation

$$
\Phi(X, Y)=C_{0,0}+C_{1,0} X+C_{0,1} Y+C_{1,1} X Y
$$


satisfying $\Phi\left(h_{1}^{(3)}(\tau), h_{4}^{(3)}(\tau)\right)=0$. Because we obtain a very simple equation, it is easy to find the coefficients $C_{i, j}$ for $0 \leq i, j \leq 1$ by substituting $q$-expansions of $h_{1}^{(3)}(\tau)$ and $h_{4}^{(3)}(\tau)$ directly. From Theorem $4(1), C_{1,0} \neq 1$ and we may assume $C_{1,0}=1$. So, $C_{1,1}=0, C_{0,1}=-2, C_{0,0}=-1$,

$$
h_{1}^{(3)}(\tau)-2 h_{4}^{(3)}(\tau)-1=0
$$

and

$$
g_{3,1}(\tau)-g_{3,-1}(\tau)=2 q g_{3,4}(\tau)
$$

It yields that

$$
a_{3}(4 n+1)=a_{3}(n)
$$

from coefficients of both sides in

$$
\frac{1}{2}\left(\sum_{n=1}^{\infty} a_{3}(n) q^{n}-\sum_{n=1}^{\infty}(-1)^{n} a_{3}(n) q^{n}\right)=q \sum_{n=1}^{\infty} a_{3}(n) q^{4 n} .
$$

\section{Proof of Theorem 2}

The process to prove Theorem 2 is similar to one done in previous section.

First, we rewrite the quotients of functions $g_{5,-1}, g_{5,1}, g_{5,2}$ and $g_{5,4}$ as Klein form products.

Lemma 7. If $g_{5, s}(\tau)$ is the function defined in $\S 1$ for $s=-1,1,2$ or 4 , then their quotients are

(1) $\frac{g_{5,1}}{g_{5,-1}}(\tau)=\zeta_{20}^{-3} \frac{K_{\frac{2}{20}} K_{\frac{5}{20}}^{8} K_{\frac{6}{20}}}{K_{\frac{1}{20}}^{2} K_{\frac{3}{20}}^{2} K_{\frac{7}{20}}^{2} K_{\frac{9}{20}}^{2} K_{\frac{10}{20}}^{2}}(\tau)$,

(2) $q \frac{g_{5,2}}{g_{5,-1}}(\tau)=\zeta_{20}^{-3} \frac{K_{\frac{2}{20}} K_{\frac{5}{20}}^{4} K_{\frac{6}{20}}}{K_{\frac{1}{20}} K_{\frac{3}{20}} K_{\frac{7}{20}} K_{\frac{9}{20}} K_{\frac{10}{20}}^{2}}(\tau)$,

(3) $q^{3} \frac{g_{5,4}}{g_{5,-1}}(\tau)=\zeta_{10}^{-3} \frac{K_{\frac{2}{20}}^{2} K_{\frac{5}{20}}^{4} K_{\frac{6}{20}} K_{\frac{1}{20}} K_{\frac{3}{20}} K_{\frac{7}{20}} K_{\frac{9}{20}} K_{\frac{10}{20}}}{(\tau)}$.

Here $K_{\frac{r}{20}}(\tau)$ is a function defined in $\S 2$ for an integer $r$ such that $0 \leq r \leq 19$.

Proof.

$$
\begin{aligned}
K_{\frac{r}{20}}(\tau) & =\prod_{j=0}^{19} \mathfrak{k}_{\left(\frac{r}{20}, \frac{j}{20}\right)(\tau)} \\
& =(-2 \pi i)^{-20} \zeta_{80}^{19 r+20} q^{\frac{r(r-20)}{40}} \prod_{n=1}^{\infty} \frac{\left(1-q^{20 n-r}\right)\left(1-q^{20 n-(20-r)}\right)}{\left(1-q^{n}\right)^{40}},
\end{aligned}
$$

we rewrite the functions in the left hand side as

$$
\frac{g_{5,1}}{g_{5,-1}}(\tau)=\prod_{n=1}^{\infty} \frac{\left(1-q^{5 n}\right)^{5}}{\left(1-q^{n}\right)} / \frac{\left(1-(-q)^{5 n}\right)^{5}}{\left(1-(-q)^{n}\right)}
$$




$$
\begin{aligned}
& =\prod_{\substack{s=2,6 \\
r=1,3,7,9}} \prod_{n=1}^{\infty} \frac{\left(1-q^{20 n-s}\right)\left(1-q^{20 n-(20-s)}\right)\left(1-q^{20 n-5}\right)^{8}\left(1-q^{20 n-15}\right)^{8}}{\left(1-q^{20 n-r}\right)^{2}\left(1-q^{20 n-(20-r)}\right)^{2}\left(1-q^{20 n-10}\right)^{4}} \\
\frac{g_{5,2}}{g_{5,-1}}(\tau) & =\prod_{n=1}^{\infty} \frac{\left(1-q^{10 n}\right)^{5}}{\left(1-q^{2 n}\right)} / \frac{\left(1-(-q)^{5 n}\right)^{5}}{\left(1-(-q)^{n}\right)} \\
& =\prod_{\substack{s=2,6 \\
r=1,3,7,9}} \frac{\left(1-q^{20 n-s}\right)\left(1-q^{20 n-(20-s)}\right)\left(1-q^{20 n-5}\right)^{4}\left(1-q^{20 n-15}\right)^{4}}{\left(1-q^{20 n-r}\right)\left(1-q^{20 n-(20-r)}\right)\left(1-q^{20 n-10}\right)^{4}}
\end{aligned}
$$

and

$$
\begin{aligned}
\frac{g_{5,4}}{g_{5,-1}}(\tau) & =\prod_{n=1}^{\infty} \frac{\left(1-q^{20 n}\right)^{5}}{\left(1-q^{4 n}\right)} / \frac{\left(1-(-q)^{5 n}\right)^{5}}{\left(1-(-q)^{n}\right)} \\
& =\prod_{\substack{s=2,6 \\
r=1,3,7,9}} \prod_{n=1}^{\infty} \frac{\left(1-q^{20 n-s}\right)^{2}\left(1-q^{20 n-(20-s)}\right)^{2}\left(1-q^{20 n-5}\right)^{4}\left(1-q^{20 n-15}\right)^{4}}{\left(1-q^{20 n-r}\right)\left(1-q^{20 n-(20-r)}\right)\left(1-q^{20 n-10}\right)^{8}} .
\end{aligned}
$$

It is omitted to compare each product because it is very tedious.

Next, we find the congruence subgroup corresponding to the function field $\mathbb{C}\left(q^{\alpha} \frac{g_{5, s}}{g_{5,-1}}\right)$ for $(\alpha, s)=(0,1),(1,2)$ and $(3,4)$. Denote $h_{1}^{(5)}(\tau)\left(\right.$ resp. $h_{2}^{(5)}(\tau)$ and $h_{4}^{(5)}(\tau)$ ) by $\frac{g_{5,1}}{g_{5,-1}}(\tau)$ (resp. $q \frac{g_{5,2}}{g_{5,-1}}(\tau)$ and $\left.q^{3} \frac{g_{5,4}}{g_{5,-1}}(\tau)\right)$.

Proposition 8. For the function $h_{1}^{(5)}(\tau), h_{2}^{(5)}(\tau)$ and $h_{4}^{(5)}(\tau)$,

$$
\mathbb{C}\left(h_{1}^{(5)}(\tau), h_{2}^{(5)}(\tau)\right) \subset A_{0}\left(\Gamma_{0}(20)\right)
$$

and

$$
\mathbb{C}\left(h_{2}^{(5)}(\tau), h_{4}^{(5)}(\tau)\right) \subset A_{0}\left(\Gamma_{0}(20)\right) .
$$

Proof. Let $\Gamma_{1}$ (resp. $\Gamma_{2}$ ) be the congruence subgroup corresponding to

$$
\mathbb{C}\left(h_{1}^{(5)}(\tau), h_{2}^{(5)}(\tau)\right)
$$

(resp. $\left.\mathbb{C}\left(h_{2}^{(5)}(\tau), h_{4}^{(5)}(\tau)\right)\right)$. Through Lemma 5 and (K5) both $\Gamma_{1}$ and $\Gamma_{2}$ contain $\Gamma(20)$. Moreover $\Gamma_{1}(20) \subset \Gamma_{1} \cap \Gamma_{2}$ because $q$ is invariant under $\left(\begin{array}{ll}1 & 1 \\ 0 & 1\end{array}\right)$ and $\Gamma_{1}(20)=\left\langle\Gamma(20),\left(\begin{array}{ll}1 & 1 \\ 0 & 1\end{array}\right)\right\rangle$. Consider that the factor $\overline{\Gamma_{0}(20)} / \overline{\Gamma_{1}(20)}$ is of order 4 and generated by the coset $\left(\begin{array}{cc}3 & 1 \\ 20 & 7\end{array}\right) \overline{\Gamma_{1}(20)}$. So we use its action on the function $K_{\frac{r}{20}}$ for $r=1,2,3,5,6,7,9$ and 10 . Let $\gamma$ be a modular action $\gamma_{7}=\left(\begin{array}{cc}3 & 1 \\ 20 & 7\end{array}\right)$. The following calculation proves that $\Gamma_{0}(20)$ is a subset of both $\Gamma_{1}$ and $\Gamma_{2}$.

$$
\left(\begin{array}{l}
K_{\frac{1}{20}} \\
K_{\frac{3}{20}} \\
K_{\frac{7}{20}} \\
K_{\frac{9}{20}}
\end{array}\right)\left(\gamma_{7} \tau\right)=(20 \tau+7)^{-20}\left(\begin{array}{cccc}
0 & \zeta_{20}^{7} & 0 & 0 \\
0 & 0 & 0 & 1 \\
\zeta_{20}^{7} & 0 & 0 & 0 \\
0 & 0 & \zeta_{5}^{-1} & 0
\end{array}\right)\left(\begin{array}{l}
K_{\frac{1}{20}} \\
K_{\frac{3}{20}} \\
K_{\frac{7}{20}} \\
K_{\frac{9}{20}}
\end{array}\right)(\tau)
$$




$$
\begin{aligned}
\left(\begin{array}{c}
K_{\frac{2}{20}} \\
K_{\frac{6}{20}}
\end{array}\right)\left(\gamma_{7} \tau\right) & =(20 \tau+7)^{-20}\left(\begin{array}{cc}
0 & \zeta_{5}^{3} \\
\zeta_{10}^{-1} & 0
\end{array}\right)\left(\begin{array}{l}
K_{\frac{2}{20}} \\
K_{\frac{6}{20}}
\end{array}\right)(\tau), \\
K_{\frac{5}{20}}\left(\gamma_{7} \tau\right) & =(20 \tau+7)^{-20} \zeta_{8}^{5} K_{\frac{5}{20}}(\tau), \\
K_{\frac{10}{20}}\left(\gamma_{7} \tau\right) & =(20 \tau+7)^{-20} \zeta_{4} K_{\frac{10}{20}}(\tau) .
\end{aligned}
$$

Hereafter we use the same notation $\Gamma_{1}$ and $\Gamma_{2}$ to point to the congruence subgroup corresponding to $\mathbb{C}\left(h_{1}^{(5)}(\tau), h_{2}^{(5)}(\tau)\right)$ and $\mathbb{C}\left(h_{2}^{(5)}(\tau), h_{4}^{(5)}(\tau)\right)$, respectively.

Proof of Theorem 2. Note that the set of inequivalent cusps for $\Gamma_{0}(20)$ is $S_{\Gamma_{0}}(20)$ $=\left\{\infty, 0, \frac{1}{2}, \frac{1}{4}, \frac{1}{5}, \frac{1}{10}\right\}$. Then we may choose the sets of inequivalent cups for $\Gamma_{1}$ and $\Gamma_{2}$ so that they are contained in $S_{\Gamma_{0}(20)}$. Like the table in proof of Proposition 6 we investigate the behaviors of the functions $K_{\frac{r}{20}}(r=1,2,3,5,6,7,9$ and 10$), h_{1}^{(5)}(\tau), h_{2}^{(5)}(\tau)$ and $h_{4}^{(5)}(\tau)$ :

\begin{tabular}{c||c|c|c|c|c|c}
\hline cusp & $\infty$ & 0 & $\frac{1}{2}$ & $\frac{1}{4}$ & $\frac{1}{5}$ & $\frac{1}{10}$ \\
\hline$K_{\frac{1}{20}}$ & $-\frac{19}{40}$ & $-\frac{133}{80}$ & $-\frac{67}{20}$ & $-\frac{67}{40}$ & $-\frac{133}{80}$ & $-\frac{59}{40}$ \\
$K_{\frac{2}{20}}$ & $-\frac{9}{10}$ & $-\frac{133}{80}$ & $-\frac{33}{20}$ & $-\frac{17}{10}$ & $-\frac{137}{80}$ & $-\frac{33}{20}$ \\
$K_{\frac{3}{20}}$ & $-\frac{51}{40}$ & $-\frac{133}{80}$ & $-\frac{67}{40}$ & $-\frac{67}{40}$ & $-\frac{137}{80}$ & $-\frac{71}{40}$ \\
$K_{\frac{5}{20}}$ & $-\frac{15}{8}$ & $-\frac{133}{80}$ & $-\frac{67}{40}$ & $-\frac{67}{40}$ & $-\frac{25}{16}$ & $-\frac{15}{8}$ \\
$K_{\frac{6}{20}}$ & $-\frac{21}{10}$ & $-\frac{133}{80}$ & $-\frac{33}{20}$ & $-\frac{17}{10}$ & $-\frac{133}{80}$ & $-\frac{37}{20}$ \\
$K_{\frac{7}{20}}$ & $-\frac{91}{40}$ & $-\frac{133}{80}$ & $-\frac{67}{40}$ & $-\frac{67}{40}$ & $-\frac{137}{80}$ & $-\frac{71}{40}$ \\
$K_{\frac{9}{20}}$ & $-\frac{99}{40}$ & $-\frac{133}{80}$ & $-\frac{67}{40}$ & $-\frac{67}{40}$ & $-\frac{133}{80}$ & $-\frac{59}{40}$ \\
$K_{\frac{10}{20}}$ & $-\frac{5}{2}$ & $-\frac{133}{80}$ & $-\frac{33}{20}$ & $-\frac{17}{10}$ & $-\frac{25}{16}$ & $-\frac{5}{4}$ \\
\hline$h_{1}^{(5)}$ & 0 & 0 & 0 & 0 & $\frac{3}{4}$ & -3 \\
$h_{2}^{(5)}$ & 1 & 0 & 0 & 0 & $\frac{1}{4}$ & -2 \\
$h_{4}^{(5)}$ & 3 & 0 & 0 & 0 & 0 & -3 \\
\hline
\end{tabular}

By Lemma 3, the widths at $\infty, \frac{1}{5}$ and $\frac{1}{10}$ are 1,4 and 1 respectively. The both widths at $\infty$ and $\frac{1}{10}$ are 1 because the width for $\Gamma_{1}$ or $\Gamma_{2}$ is a positive integer less than one for $\Gamma_{0}(20)$ at same cusp. Let $h$ be the width at $\frac{1}{5}$ for $\Gamma_{1}$ or $\Gamma_{2}$. Since 4 is the one at $\frac{1}{5}$ for $\Gamma_{0}(20), 1 \leq h \leq 4$. The fact that $h_{2}^{(5)}(\tau)$ is the element of $A_{0}\left(\Gamma_{1}\right)$ and $A_{0}\left(\Gamma_{2}\right)$ means that

$$
q_{h}^{n_{0}}=\left(q^{\frac{1}{h}}\right)^{n_{0}}=q^{\frac{1}{4}}
$$

if we write an integer $n_{0}$ as the order at $\frac{1}{5}$ with considering its width. Hence $h$ must be 4 . Therefore with regards to width at each cusps,

\begin{tabular}{c||c|c|c|c|c|c}
\hline cusp & $\infty$ & 0 & $\frac{1}{2}$ & $\frac{1}{4}$ & $\frac{1}{5}$ & $\frac{1}{10}$ \\
\hline$h_{1}^{(5)}$ & 0 & 0 & 0 & 0 & 3 & -3 \\
$h_{2}^{(5)}$ & 1 & 0 & 0 & 0 & 1 & -2 \\
$h_{4}^{(5)}$ & 3 & 0 & 0 & 0 & 0 & -3 \\
\hline
\end{tabular}


we find the equation of them. For the convenience, write $h_{1}^{(5)}$ (resp. $h_{2}^{(5)}$ and $\left.h_{4}^{(5)}\right)$ as $h_{1}$ (resp. $h_{2}$ and $h_{4}$ ).

Through Theorem 4 we get two equations:

$$
\begin{gathered}
h_{1}^{2}-2 h_{1} h_{2}-h_{1}-4 h_{2}^{3}=0, \\
h_{2}^{3}-2 h_{2} h_{4}-4 h_{4}^{2}-h_{4}=0
\end{gathered}
$$

By adding four times of (4.1) to (4.2), we obtain that

$h_{1}^{2}-16 h_{4}^{2}-4 h_{2}-h_{1}-8 h_{2} h_{4}-2 h_{1} h_{2}=\left(h_{1}+4 h_{4}\right)\left(h_{1}-4 h_{4}-1+2 h_{2}\right)=0$.

$\left(h_{1}+4 h_{4}\right)(\tau)$ is a modular function with respect to $\Gamma_{0}(20)$ and $\left(h_{1}+4 h_{4}\right)\left(\frac{1}{5}\right) \neq 0$ means that it is a nonzero modular function. So it might have only finite zeros. Hence the modular function $h_{1}-4 h_{4}-1+2 h_{2}$ have infinitely many zeros in $\mathfrak{H}$ and it is identically zero.

$$
\begin{aligned}
& h_{1}(\tau)-4 h_{4}(\tau)-1+2 h_{2}(\tau)=0 \\
\Leftrightarrow & g_{5,1}(\tau)-g_{5,-1}(\tau)+2 q g_{5,2}(\tau)=4 q^{3} g_{5,4}(\tau) \\
\Leftrightarrow & \sum_{n=1}^{\infty} a_{5}(n) q^{n}+\sum_{n=1}^{\infty}(-1)^{n} a_{5}(n) q^{n}+2 \sum_{n=1}^{\infty} a_{5}(n) q^{2 n+1}=4 \sum_{n=1}^{\infty} a_{5}(n) q^{4 n+3} .
\end{aligned}
$$

Hence our second theorem is proved.

Acknowledgements. We are grateful to the referee for several helpful suggestions on the improvement of this paper.

\section{References}

[1] N. Baruah and B. C. Berndt, Partition identities and Ramanujan's modular equations, J. Combin. Theory Ser. A 114 (2007), no. 6, 1024-1045.

[2] A. Berkovich and H. Yesilyurt, New identities for 7-cores with prescribed BG-rank, Discrete Math. 308 (2008), no. 22, 5246-5259.

[3] B. C. Berndt, Ramanujan's Notebooks, Part III, Springer-Verlag, New York, 1991.

[4] B. Cho, J. K. Koo, and Y. K. Park, Arithmetic of the Ramanujan-Göllnitz-Gordon continued fraction, J. Number Theory 129 (2009), no. 4, 922-947.

[5] F. Garvan, D. Kim, and D. Standon, Cranks and t-cores, Invent. Math. 101 (1990), no. $1,1-17$.

[6] M. D. Hirschhorn and J. A. Sellers, Elementary proofs of various facts about 3-cores, Bull. Aust. Math. Soc. 79 (2009), no. 3, 507-512.

[7] N. Ishida and N. Ishii, The equations for modular function fields of principal congruence subgroups of prime level, Manuscripta Math. 90 (1996), no. 3, 271-285.

[8] B. Kim, On inequalities and linear relations for 7-core partitions, Discrete Math. 310 (2010), no. 4, 861-868

[9] D. Kubert and S. Lang, Modular Units, Springer-Verlag, New York-Berlin, 1981.

[10] S. Ramanujan, Notebooks (2 volumes), Tata Institute of Fundamental Research, Bombay, 1957.

School of Mathematics

Korea Institute for Advanced Study

SeOul 130-722, Korea

E-mail address: ykpark@math.kaist.ac.kr 